\title{
Creencias asociadas al consumo y dependencia de sustancias psicoactivas ${ }^{*}$
}

\author{
Beliefs associated with consumption and dependence on \\ psychoactive substances
}

Crenças associadas ao consumo e dependência de substâncias psicoativas

\author{
Ángela Vargas Vargas ${ }^{* *}$ \\ Nora Helena Londoño Arredondo
}

Recibido 29. 03. 2016 • Arbitrado 26. 04. 2016 • Aprobado 13.05. 2016

\section{Resumen}

Antecedentes: el estudio de las adicciones a sustancias psicoactivas ha sido un tema de interés en la agenda pública a lo largo de la historia dado su prevalencia e implicaciones en la salud mental y en el ámbito social y legal. La tesis central del estudio se concreta en que las creencias nucleares predisponen la dependencia al consumo de sustancias psicoactivas, hallándose variables intervinientes como la depresión y la ansiedad que complejizan la dependencia y el craving (ansia) de los individuos. El objetivo de esta investigación fue establecer las creencias que mejor se asocian de manera predictiva con el consumo y dependencia de sustancias psicoactivas. Método: estudio de casos y controles, participaron 80 personas, 35 en el grupo casos intervenidos

Artículo presenta resultados de la investigación realizada para otra el título de magíster en psicología, Universidad de San Buenaventura, Medellín, Colombia, 2013-2015.

* Psicóloga, Universidad de San Buenaventura, Medellín; Especialista en Terapia Cognitiva, Universidad de San Buenaventura, Medellín; Magíster en psicología, Universidad der San Buenaventura, Medellín, Docente Universidad de San Buenaventura, Medellín, Colombia. Contacto: algena-vargas@hotmail.com

*** Psicóloga y Magíster en Psicología de la Universidad de San Buenaventura, Medellín; Especialista en psicología clínica y Doctora en Psicología de la Universidad del Norte, Barranquilla; Docente Universidad de San Buenaventura, Medellín, Colombia. Contacto: nora.londono@usbmed.edu.co 
en Comunidad terapéutico, y 45 en el grupo control. Instrumentos: escala de creencias acerca del abuso de sustancias (Wright, 1999), CES-D (Redloff \& Loche, 1986), SCL-90-R (Derogatis, 1996), MINI (Scheehan \& Lecrubier, 1998). Resultados: el primer factor explica el 19,195\% de la VE y los demás hasta un $8 \%$. Estos factores se refieren a los siguientes categorías: Categoría 1: creer que las sustancias psicoactivas son necesarias para vivir ( 5 ítems, $\mathrm{VE}=19,195$ ), Categoría 2: creer que las drogas me controlan ( 4 ítems, VE=12,663), categoría 3: creer que las sustancias psicoactivas aumentaran el poder social y físico (3 ítems, $\mathrm{VE}=11,555$ ), categoría 4: creer que el consumo de sustancias psicoactivas tiene un origen fuera de control ( 3 ítems, VE= 10,649), Categoría 5: creer que el consumo de sustancias psicoactivas no es problemático (3 ítems, VE=8,908), Categoría 6: Valoración negativa de sí mismo (2 ítems, VE=8,743). Conclusiones: las creencias influyen significativamente y de manera predictiva al consumo y la dependencia de sustancias psicoactivas. Más que la depresión y la ansiedad, las creencias tienen un mayor nivel de predicción para el consumo y dependencia de sustancias psicoactivas.

Palabras clave: Sustancias psicoactivas; creencias; dependencia; depresión; ansiedad.

\section{Abstract}

Background: the study of addictions to psychoactive substances has been a topic of interest on the public agenda throughout history due to its prevalence and implications of mental health and the social and legal field. The central thesis of the study is specific in that the core beliefs predispose dependence to psychoactive substances, being intervening variables such as depression and anxiety complicate dependence and craving of individuals. The objective of this research was to establish the belief that better explain the use and dependence of psychoactive substances. Method: case-control study, involving 80 people, 35 in the cases operated in therapeutic community, and 45 in the control group. Instruments: Scale of beliefs about substance abuse 
(Wright, 1999), CES-D (Radloff \& Loche, 1986) SCL-90-R (Derogatis, 1996), MINI (Scheehan \& Lecrubier, 1998). Results: The first factor explains $19,195 \%$ of the VE and the other to $8 \%$. These relate to the following categories: Category 1: Believing that psychoactive substances are necessary to live ( 5 items, $\mathrm{VE}=19,195$ ), Category 2: Believe me that drugs control ( 4 items, $\mathrm{VE}=12,663$ ), Category 3 : Believing that psychoactive substances increase social and physical power (3 items, $\mathrm{VE}=11,555$ ), Category 4: Believing that the use of psychoactive substances has an origin out of control (3 items, VE $=10,649$ ), Category 5: Believing the use of psychoactive substances is not problematic (3 items, VE $=8,908$ ), Category 6: Negative Rating himself (2 items, VE = 8.743). Conclusions: beliefs significantly influence and predictively consumption and dependence on psychoactive substances. More than depression and anxiety, beliefs have a higher level of prediction for the use and dependence of psychoactive substances.

Keywords: Psychoactive substances; beliefs; dependence; depression; anxiety.

\section{Resumo}

O estudo da dependência de substâncias psicoativas tem sido, ao longo da história, um assunto de interesse na agenda pública por causa da sua prevalência e suas implicações tanto para a saúde mental, como no campo social e legal. A tese central do estudo assenta na premissa de que que as crenças centrais predispõem a dependência de substâncias psicoactivas, sendo variáveis intervenientes na depressão e ansiedade e que complicam a dependência e o desejo dos indivíduos. O objetivo deste trabalho foi examinar quais crenças que melhor se associam preditivamente ao consumo e dependência de substâncias psicoativas. Método: estudo caso-controle, envolvendo 80 indivíduos, 35 fazendo parte do grupo dos casos operados em comunidade terapêutica e 45 no grupo de controlo. Instrumentos: Escala de crenças sobre abuso de substâncias (Wright, 1999), CES-D (Redloff \& Loche, 1986), SCL-90-R (Derogatis, 1996), MINI (Scheehan e Lecrubier, 1998). Resultado: O 
primeiro factor explica $19,195 \%$ do VE e o outro a $8 \%$. Esses fatores se relacionam com as seguintes categorias: Categoria 1: Crer que as substâncias psicoativas são necessárias para viver ( 5 itens, VE $=19.195$ ), Categoria 2: Crer que as drogas me controlam ( 4 itens, VE $=12.663$ ), Categoria 3: Crer que as substâncias psicoativas aumentam o poder social e físico ( 3 itens, $\mathrm{VE}=11.555$ ), Categoria 4: Crer que o uso de substâncias psicoativas tem uma origem fora de controle (3 itens, VE = 10.649), Categoria 5: Crer que o uso de substâncias psicoativas não é problemático (3 itens, $\mathrm{VE}=8908$ ), Categoria 6: Avaliação negativa de si mesmo ( 2 itens, $\mathrm{VE}=8,743$ ). Conclusões: as crenças influenciam significativamente o consumo e dependência de substâncias psicoativas. As crenças são melhor preditoras do consumo e dependência de substâncias psicoativas do que a depressão e ansiedade.

Palavras-chave: Substâncias psicoativas; crenças; dependência; depressão; ansiedade.

\section{Introducción}

El abuso de sustancias psicoactivas es un problema social y legal grave; se considera que la utilización de drogas ilegales puede ser responsable de un gran número de crímenes violentos, homicidios, problemas de salud y en general dificultades sociales (Beck, Wright, Newman \& Liese, 1999), y se encuentra asociado frecuentemente con otros trastornos mentales como la depresión y la ansiedad (Asociación Psiquiátrica Americana [APA], 2013).

La Organización Mundial de la Salud (OMS), en mayo del 2010, aprobó por primera vez una Estrategia global para la reducción del consumo nocivo de alcohol y el Consejo Directivo de la Organización Panamericana de la Salud (OPS) adoptó en septiembre del 2010, también por primera vez, una estrategia sobre el uso de sustancias y la salud pública. En Octubre de 2010, la OMS y la Organización Panamericana de la Salud, en el denominado Consenso de Panamá declararon que los trastornos mentales, por uso de sustancia psicoactiva, signifi- 
can una gran carga en relación a la morbilidad, mortalidad y discapacidad, anudado a la poca atención que impide el acceso, diagnósticos y tratamiento adecuado de los afectados.

La prevalencia mundial del consumo de drogas psicoactivas es de $3,3 \%$ al 6,1\% entre personas de 15 a 64 años de edad (APA, 2013). En el contexto colombiano, en población de 18 a 65 años de edad, la prevalencia de consumidores de bebidas alcohólicas es del 94\%, 48,1\% de cigarrillo, $12,7 \%$ de medicamentos no formulados, $10,8 \%$ marihuana, y $4 \%$ cocaína. El inicio del abuso de sustancias se ha asociado con la aparición de trastornos psiquiátricos, identificándose éstos como factor de riesgo (Torres, Bareño y Sierra, 2012).

Según el Ministerio de Salud y Colciencias (2015), en la encuesta Nacional de Salud Mental (2015) realizada en Colombia, "se encuentra mayor proporción de consumo en hombres, y la sustancia ilegal más consumida es la marihuana, sin embargo, el alcohol y el cigarrillo siguen siendo los más consumidos" (p.162), en lo relacionado con sustancias legales es más frecuente el consumo de alcohol que de cigarrillo.

Dentro de los resultados asociados al consumo de sustancias, se destaca que en los adolescentes el consumo de alcohol en exceso es de $5,2 \%$ y abuso es de 2,8 \%. En lo que refiere a las sustancias ilegales, el $3,5 \%$ de los adolescentes refiere haber consumido marihuana en algún momento en la vida, de los cuales el 48,8 \% lo han hecho en los últimos 12 meses. La población colombiana entre 18 y 44 el 15,9 \% de la población ha consumido cigarrillo o algún derivado del tabaco en la vida. De las sustancias con potencial de abuso y dependencia, se encuentra que el $6,3 \%$ de la población reporta haber consumido canabiniodes. La población de 45 años en adelante las prevalencias del consumo son el $2,6 \%$ de canabinoides, el o,8\% ha consumido bazuco alguna vez en la vida y 0,7 \% de opioides (Ministerio de Salud y Colciencias, 2015).

En relación al impacto por el consumo de sustancias, la Encuesta Nacional de Salud Mental refiere:

Los consumidores de cannabis las comorbilidades con estrés postraumático, en los últimos 12 meses, y en la vida, haber presentado fobia social en algún mo- 
mento y trastorno de ansiedad generalizada en los últimos 12 meses. En las personas con consumo de otras sustancias psicoactivas hay una mayor prevalencia de (...), trastorno de estrés postraumático (Ministerio de Salud y Colciencias, 2015, p.162).

La Asociación Psiquiátrica Americana (APA, 2013) distingue entre abuso de sustancia y dependencia de la misma. El abuso se define como un patrón disfuncional de utilización de sustancias psicoactivas mientras que la dependencia es considerada algo más serio que el abuso, se define como "incapacidad de control del consumo" (es decir, adicción fisiológica).

Factores sociales, ambientales y de personalidad influyen en el uso de sustancias y va más allá de las propiedades farmacológicas simples de estos fármacos. El ambiente social puede influir en el consumo de drogas o alcohol. Por ejemplo, el alcoholismo tiene mucha prevalencias en algunos grupos étnicos y prácticamente es inexistente en otros, como en los mormones, que requieren la abstinencia antes de que la persona entre a formar parte del grupo. Otros grupos sociales pueden condicionar la aceptación del miembro al grupo a que utilicen drogas o beban. Los soldados en Vietnam utilizaron mucho las drogas ilegales pero, en su mayoría, abandonaron dicho hábito fuertemente arraigado después de volver a su hogar (Beck et al.., 1999).

Factores sociales y personales al igual que déficits en habilidades sociales y académicas, baja autoestima, ansiedad y estrés se articulan y pueden facilitar la iniciación del consumo de alcohol y drogas. Los estudios realizados en Estados Unidos han mostrado que los trastornos mentales usualmente preceden a los del abuso de sustancias psicoactivas. Entre el principio de la patología mental y el de un trastorno por el uso de sustancias hay un intervalo, en promedio de 5 años (García, Rodríguez \& Córdova, 2008).

Las variables demográficas (genero, edad, ocupación y religión) tienen algún valor predictivo sobre la probabilidad de consumo de sustancias psicoactivas, y el consumo de alcohol es un predictor del consumo de otras (Ministerio del Interior y de Justicia, Ministerio de la Protección Social y Dirección Nacional de Estupefacientes, 2009). Además, la frecuencia del consumo de drogas se ha identificado ma- 
yor en fumadores que consumen más de 2 paquetes de cigarrillos al día comparado con fumadores con menor consumo de cigarrillos ( $\mathrm{Su}$, Kung, Huang \& Lee, 2011).

Siendo de tanta importancia la identificación de los factores de riesgo para abordarlos de manera preventiva en los diferentes niveles de intervención, se ha considerado la necesidad de indagar sobre los factores de riesgo específicos a los estilos de pensamientos asociados con el consumo, abuso y dependencia de sustancias psicoactivas. Así, en los estudios sobre el impacto de las creencias en la conducta humana, el estudio realizado en el 2011 en Asia, refiere que los pacientes de sexo masculino tienen creencias relativamente más fuertes sobre el consumo de drogas (Su et al., 2011). Sin embargo, no se encuentran estudios que relacionen creencias específicas a los trastornos por abuso de sustancias psicoactivas, y que, además, analicen el impacto de indicadores clínicos como la depresión y la ansiedad en dicha asociación. Un antecedente relevante es el aporte de Wright, quien abordó las creencias asociadas con la drogodependencia y construyo la prueba de creencias (Beck et al., 1999). Sin embargo, después de su muerte, no se han generado estudios posteriores relacionados.

Apoyados en lo anterior, se pretende aportar al conocimiento de las adicciones a través del estudio sobre las creencias asociadas a la dependencia de sustancias y factores intervinientes como la depresión y la ansiedad.

\section{Método}

La investigación fue de corte cuantitativo, empírico-analítico de casos y controles: se observó un evento en un tiempo específico (estudio de corte transversal), analizando y buscando explicaciones a factores relacionados entre sí, de modo tal que se logró establecer cuáles variables se asociaban significativamente.

\section{Participantes}

La investigación se realizó eligiendo una población objeto de estudio perteneciente a una comunidad terapéutica del área metropolitana 
de Medellín (Colombia), los cuales recibían tratamiento por el abuso de sustancias psicoactivas, y un grupo control. La selección de la muestra fue no aleatoria, participaron 80 personas, el grupo caso se conformó por 35 personas ( 27 hombres, 8 mujeres); que se encontraban en un proceso de rehabilitación por abuso de sustancias psicoactivas, mientras que el grupo control estuvo compuesto por 45 personas (19 hombres, 26 mujeres), no abusadoras de sustancias psicoactivas.

\section{Instrumentos}

La escala de creencias acerca del abuso de sustancias (Beck, Wright, Newman \& Liese, 1999): autoinforme compuesto de 20 ítems. Una puntuación de (1) indica que la persona está totalmente desacuerdo con dicha sentencia. Una puntuación de (7) indica que la persona está totalmente de acuerdo con dicha sentencia. Esta escala mide muchas de las creencias más frecuentes acerca de la utilización de las drogas.

La escala de Depresión del Centro de Estudios Epidemiológicos, Center for Epidemiologic Studies Depression Scale (CES-D; Radloff \& Locke, 1986) fue otro instrumento usado para identificar trastornos depresivos en población general. En su versión original la CES-D consta de 20 ítems, cada uno de los cuales corresponde a un síntoma habitual y representativo del trastorno depresivo. La persona evalúa cada ítem en función de la frecuencia en que lo ha experimentado en el último mes, con una puntuación de 1 a 4, donde 1 significa prácticamente nunca, 2 algunas veces, 3 bastantes veces, 4 casi todo el tiempo.

Inventario de Síntomas de Derogatis, Revisado (Derogatis Symptom Checklist, Revised [SCL-90-R]; Derogatis, 2002): es un instrumento que se usó para evaluar el grado de malestar psicológico actual [distress2] que experimenta una persona. Consiste en un listado de 90 síntomas psiquiátricos de variado nivel de gravedad, frente a los cuales el respondiente debe indicar en qué medida lo han molestado o perturbado cada uno de esos problemas durante el período comprendido entre el día de la evaluación y la semana inmediatamente anterior. 
Para responder, se ofrece una escala de cinco opciones acotada por los términos "nada", "muy poco", "poco", "bastante” y "mucho", que en la corrección es valorada en un rango de o a 4 puntos.

Mini International Neuropsychiatric Interview (M.I.N.I) (Scheehan \& Lecrubier, 1998): Es una entrevista neuropsiquiátrica internacional dividida en módulos, se presenta en un recuadro gris una o varias preguntas (filtro) correspondientes a los criterios diagnósticos principales del trastorno. Para esta investigación se utilizaron los módulos correspondientes al abuso y dependencia de alcohol y el módulo de trastornos asociados al uso de sustancias psicoactivas no alcohólicas.

\section{Procedimiento}

Se solicitó el consentimiento informado de cada uno de los participantes de la investigación, tanto control, como casos, enfatizando en el derecho al anonimato y que solo se usarían los datos para los fines de la investigación. Se realizó la entrevista estructurada MINI por parte de los investigadores y de manera individual los participantes contestaron los cuestionarios en presencia de los investigadores quienes estuvieron atentos de responder las inquietudes.

Se determinaron las principales creencias acerca del abuso de sustancias asociadas a los trastornos por el consumo de sustancias psicoactivas a través de análisis comparativos entre los grupos casos y controles y análisis de regresión lineal. Los datos de la investigación fueron procesados con el "Paquete Estadístico para las Ciencias Sociales” (Statistical Package for Social Sciences, SPSS) versión 22.0 para Windows.

\section{Resultados}

\section{Análisis de confiabilidad de las escalas}

El análisis de fiabilidad de la escala de creencias acerca del abuso de sustancias con la prueba Alfa de Cronbach obtuvo un nivel general alto $(0,84)$, el alfa de Cronbach para la escala de Depresión del Centro 
de Estudios Epidemiológicos y el Inventario de Síntomas de Derogatis, Revisado fue de 0,905 y 0,897 respectivamente; estos datos confirmaron la pertinencia de los instrumentos aplicados.

\section{Análisis descriptivo del consumo de sustancias}

La frecuencia en el consumo de sustancia legal e ilegal, se detallan en la tabla 1; se destaca por obvias razones, el predominio del consumo, abuso y dependencia de sustancias legales e ilegales en el grupo de casos.

Tabla 1. Frecuencia de consumo de sustancias de los grupos casos $y$ controles

\begin{tabular}{|lcccc|}
\hline \multirow{2}{*}{ Variables } & \multicolumn{2}{c}{ Casos (n=35) } & \multicolumn{2}{c|}{ Controles (n=45) } \\
\cline { 2 - 5 } & Frecuencia & \% & Frecuencia & \% \\
\hline Alcohol & & & & \\
\hline Consumo & 24 & 68,6 & 12 & 26,7 \\
\hline Abuso & 19 & 54,3 & 5 & 11,1 \\
\hline Dependencia & 18 & 51,4 & 3 & 6,7 \\
\hline Sustancias & & & & 13,3 \\
\hline Consumo & 33 & 94,3 & 6 & 6,7 \\
\hline Abuso & 32 & 91,4 & 3 & 4,4 \\
\hline Dependencia & 32 & 91,4 & 2 & \\
\hline
\end{tabular}

Fuente: Autores

\section{Análisis factorial exploratorio del cuestionario de creencias}

Se realizó el análisis factorial del cuestionario de creencias con rotación Varimax con normalización Kaiser, el cual reportó 6 factores, con valores eigen superiores a 1 que acumularon el 71,714\% de la varianza total (VE) (tabla 2). 
Tabla 2. Categorización de las creencias

\begin{tabular}{|lccc|}
\hline Categoría & Valores eigen & \% Varianza & \% Acumulado \\
\hline 1 & 3,839 & 19,195 & 19,195 \\
\hline 2 & 2,533 & 12,663 & 31,858 \\
\hline 3 & 2,311 & 11,555 & 43,413 \\
\hline 4 & 2,130 & 10,649 & 54,063 \\
\hline 5 & 1,782 & 8,908 & 62,970 \\
\hline 6 & 1,749 & 8,743 & 71,714 \\
\hline
\end{tabular}

Fuente: Autores

El primer factor explica el 19,195\% de la VE y los demás hasta un 8\%. Estos factores se refieren a los siguientes categorías: categoría 1: creer que las sustancias psicoactivas son necesarias para vivir ( 5 ítems, $\mathrm{VE}=19,195$ ), categoría 2: creer que las drogas me controlan (4 ítems, $\mathrm{VE}=12,663$ ), categoría 3: creer que las sustancias psicoactivas aumentaran el poder social y físico ( 3 ítems, VE=11,555), categoría 4: creer que el consumo de sustancias psicoactivas tiene un origen fuera de control (3 ítems, VE= 10,649), categoría 5: creer que el consumo de sustancias psicoactivas no es problemático ( 3 ítems, VE=8,908), categoría 6: valoración negativa de sí mismo (2 ítems, $\mathrm{VE}=8,743$ ).

En la matriz de componente rotado (tabla 3), se detallan los elementos que permiten expresar las variables originales como una combinación lineal de los componentes principales; el método empleado fue el de extracción, análisis de componentes principales. 
Tabla 3. Matriz de componente rotado

\begin{tabular}{|c|c|c|c|c|c|c|}
\hline & $\begin{array}{c}\text { Factor } \\
1\end{array}$ & $\begin{array}{c}\text { Factor } \\
2\end{array}$ & $\begin{array}{c}\text { Factor } \\
3\end{array}$ & $\begin{array}{c}\text { Factor } \\
4\end{array}$ & $\begin{array}{c}\text { Factor } \\
5\end{array}$ & $\begin{array}{c}\text { Factor } \\
6\end{array}$ \\
\hline $\begin{array}{l}\text { 1. La vida sin tomar } \\
\text { drogas es aburrida }\end{array}$ & & & & & ,509 & \\
\hline $\begin{array}{l}\text { 2. Tomar drogas es la } \\
\text { única forma de que } \\
\text { pueda incrementar mi } \\
\text { creatividad y produc- } \\
\text { tividad }\end{array}$ & & & 805 & & & \\
\hline $\begin{array}{l}\text { 3. No puedo funcionar } \\
\text { sin ellas }\end{array}$ & 856 & & & & & \\
\hline $\begin{array}{l}\text { 4. Es la única forma de } \\
\text { afrontar el dolor de mi } \\
\text { vida }\end{array}$ & 666 & & & & & \\
\hline $\begin{array}{l}\text { 5. No estoy preparado } \\
\text { para dejar de tomar } \\
\text { drogas }\end{array}$ & & & &, 511 & & \\
\hline $\begin{array}{l}\text { 6. Los síntomas de abs- } \\
\text { tinencia y los impulsos } \\
\text { hacen que tome drogas. }\end{array}$ & & & & & ,423 & \\
\hline $\begin{array}{l}\text { 7. Mi vida no mejorará, } \\
\text { incluso aunque deje de } \\
\text { tomar drogas. }\end{array}$ &, 504 & & & & & \\
\hline $\begin{array}{l}\text { 8. La única forma de } \\
\text { manejar mi ira es usan- } \\
\text { do drogas. }\end{array}$ & 801 & & & & & \\
\hline $\begin{array}{l}\text { 9. La vida sería de- } \\
\text { primente si dejara las } \\
\text { drogas. }\end{array}$ & 819 & & & & & \\
\hline $\begin{array}{l}\text { 10. No merezco recupe- } \\
\text { rarme de las drogas. }\end{array}$ & & & & & & 833 \\
\hline $\begin{array}{l}\text { 11. No soy una persona } \\
\text { suficientemente fuerte } \\
\text { como para dejarlo. }\end{array}$ & & ,705 & & & & \\
\hline
\end{tabular}


12. No podría ser social sin tomar drogas.

13. El abuso de sus-

tancias no resulta un

problema para mí.

14. El síndrome de abstinencia y los impulsos no desaparecerán a menos que tome , 638 drogas.

15. Mi abuso de sustancias lo provoca otra persona (p. ej., mi esposa, novio o novia, , 651 o un miembro de la familia).

16. Si alguien tiene un problema con las drogas, eso es algo completamente genético.

17. No me puedo relajar sin las drogas.

18. Tener estos problemas con las drogas significa que básicamente soy una mala persona. 19. No puedo controlar mi ansiedad sin tomar drogas.

20. No puedo divertirme en esta vida a menos que tome drogas.

Fuente: Autores

\section{Análisis comparativos de los grupos}

Se realizaron análisis comparativos de los grupos con relación a las variables de análisis, y se encontraron diferencias estadísticamente significativas en las puntuaciones de creencia, depresión y ansiedad del 
grupo casos al compararlas con el grupo control ( $\mathrm{p}<0,050$ con la prueba U de Mann-Whitney) (tabla 4); Creencias sobre el abuso de sustancias (U Mann-Whitney=238,00o, $\mathrm{p}=0,000$ ); Ansiedad promediada (U Mann-Whitney $=342,500, p=0,000$ ); Ansiedad fóbica promediada (U Mann-Whitney=558,000, p=0,17);y depresión (U Mann-Whitney $=566,500, p=0,35)$.

Tabla 4. Análisis comparativo de las variables clínicas de los grupos casos $y$ controles

\begin{tabular}{|lcccc|}
\hline \multicolumn{1}{|c}{ Variables } & Casos & Controles & $\mathbf{P}$ & $\begin{array}{c}\text { U de } \\
\text { Mann-Whitney }\end{array}$ \\
\hline & $\begin{array}{c}\text { Media (des- } \\
\text { viación) }\end{array}$ & $\begin{array}{c}\text { Media } \\
\text { (desviación) }\end{array}$ & & \\
\hline $\begin{array}{l}\text { Creencias sobre el abuso } \\
\text { de sustancias }\end{array}$ & $40,7(17,6)$ & $24,9(10,03)$ & , 000 & 238,000 \\
\hline Ansiedad promedia & $1,2(0,91)$ & $0,5(0,75)$ & ,000 & 342,500 \\
\hline $\begin{array}{l}\text { Ansiedad fóbica } \\
\text { promediada }\end{array}$ & $0,55(0,69)$ & $0,22(0,48)$ &, 017 & 558,000 \\
\hline Depresión & $23,7(16,1)$ & $15,3(7,92)$ &, 035 & 566,500 \\
\hline
\end{tabular}

Fuente: Autores

\section{Análisis de regresión}

Se realizaron los análisis de regresión, estableciendo como variable dependiente el grado de dependencia de sustancias y como variables independientes las creencias acerca del consumo de sustancias, la variable clínica de ansiedad (general y fóbica) y de depresión. Los resultados reportaron un solo nivel de predicción para la dependencia de sustancias, con la variable Creencias acerca del consumo. El método empleado fue por pasos (criterios: probabilidad de F para entrar $<=$ ,050, probabilidad de F para eliminar $>=, 100$ ). Las creencias acerca del consumo de sustancias predice el posterior grado de dependencia a las mismas (Beta $=, 468 ; \mathrm{p}=, 000)$ 
Tabla 5. Análisis de regresión

\begin{tabular}{|c|c|c|c|c|c|c|c|c|c|c|}
\hline \multicolumn{11}{|c|}{ Coeficientes $^{\mathrm{a}}$} \\
\hline \multirow[t]{2}{*}{ Modelo } & \multicolumn{2}{|c|}{$\begin{array}{l}\text { Coeficientes } \\
\text { no estandari- } \\
\text { zados }\end{array}$} & \multirow{2}{*}{$\begin{array}{l}\begin{array}{l}\text { Coeficientes } \\
\text { estandari- } \\
\text { zados }\end{array} \\
\qquad \underset{0}{\pi}\end{array}$} & \multirow[t]{2}{*}{$\mathrm{t}$} & \multicolumn{3}{|c|}{ Sig. $\begin{array}{c}95,0 \% \text { intervalo } \\
\text { de confianza } \\
\text { para B }\end{array}$} & \multicolumn{3}{|c|}{ Correlaciones } \\
\hline & B & 密 & & & & 营泀苛 & 总泀 & 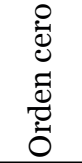 & . & \begin{tabular}{l}
$\stackrel{ \pm}{ \pm}$ \\
\multirow{\sigma}{*}{}
\end{tabular} \\
\hline (Constante) &,- 043 & ,112 & &,- 381 & ,704 &,- 265 & ,179 & & & \\
\hline $\begin{array}{l}\text { Creencias } \\
\text { sobre } \\
\text { abuso de } \\
\text { sustancias }\end{array}$ & ,015 & ,003 & ,468 & $\begin{array}{l}4,67 \\
8\end{array}$ & , 000 & ,008 & ,021 & ,468 & & ,468 \\
\hline
\end{tabular}

Nota: a. Variable dependiente: dependencia de sustancias

Fuente: elaboración propia

\section{Discusión}

Las creencias asociadas al consumo de sustancias se agruparon en 6 categorías, que se consideran creencias nucleares que mantienen la conducta adictiva. Según Beck et al. (1999) "las creencias se definen como estructuras cognitivas relativamente rígidas y duraderas, que no son fáciles de modificar por la experiencia” (p.231).

La primera categoría de creencias agrupan la creencia nuclear creer que las sustancias psicoactivas son necesarias para vivir, evidencia la necesidad primaria para consumir sustancia psicoactivas, en tanto se les considera indispensables para la vida. Las creencias que comprenden esta creencia nuclear son "no puedo funcionar sin ellas", "es la única forma de afrontar el dolor de mi vida", "mi vida no mejorará, incluso aunque deje de tomar drogas", "la única forma de manejar mi ira es usando drogas, "la vida sería deprimente si dejara las drogas". Creer que las sustancias psicoactivas son necesarias para vivir consolida las razones cognitivas para que los individuos se mantengan en 
el círculo viciosos el consumo, la droga consigue controlar a los individuos adictos, sus objetivos, valores y sus lazos de unión pasan a estar subordinados a la utilización de las sustancias; no logrando controlar su vida con eficacia (Beck et al., 1999).

La segunda categoría de creencias acerca del abuso de sustancias que se asocian con el consumo de sustancias psicoactivas, es la creencia nuclear de creer que las drogas me controlan. Las creencias que integran esta creencia nuclear son: "no soy una persona suficientemente fuerte como para dejarlo", "el síndrome de abstinencia y los impulsos no desaparecerán a menos que tome drogas", "tener estos problemas con las drogas significa que básicamente soy una mala persona, "No puedo controlar mi ansiedad sin tomar drogas". Esta creencia nuclear predice los niveles de craving, de ahí que sea tan complejo disminuir que aparezca el deseo de consumo (Martínez, Verdejo \& Becoña, 2012).

La tercera categoría consolida la creencia nuclear creer que las sustancias psicoactivas aumentaran el poder social y físico. Las creencias que componen esta creencia nuclear se centran en "tomar drogas es la única forma de que pueda incrementar mi creatividad y productividad", "no me puedo relajar sin las drogas", y "no puedo divertirme en esta vida a menos que tome drogas". Mantener este tipo de creencia nuclear posibilita la necesidad imperante del consumo, el cual va a mediar las relaciones del individuo, buscando contrarrestar la sensación de indefensión.

La cuarta creencia nuclear analizada fue creer que el consumo de sustancias psicoactivas tiene un origen fuera de control. Esta creencia nuclear la conforman "no estoy preparado para dejar de tomar drogas", "mi abuso de sustancias lo provoca otra persona (p. ej., mi esposa, novio o novia, o un miembro de la familia), "si alguien tiene un problema con las drogas, eso es algo completamente genético". Esta creencia ratifica la idea de que los individuos adictos no tienen apenas o nada de control sobre sus impulsos y conducta, por esto el craving es irresistible (Beck et al., 1999). 
La quinta creencia nuclear es creer que el consumo de sustancias psicoactivas no es problemático. La conforman creencias como "la vida sin tomar drogas es aburrida", "los síntomas de abstinencia y los impulsos hacen que tome drogas" y "el abuso de sustancias no resulta un problema para mí", son las creencias que componen la creencia nuclear. Esta creencia supone una egosintonía con el consumo de sustancias. Los individuos suelen ignorar o minimizar los problemas derivados del consumo o atribuirlos a causas externas. Un factor importante a la hora de mantener esta dependencia es la abstinencia de la sustancia le producirá efectos intolerable. (Beck et al., 1999).

La última creencia nuclear es la valoración negativa de sí mismo, el cual se explica con las creencias del tipo "no merezco recuperarme de las drogas", "no podría ser social sin tomar drogas". Esta creencia el énfasis se acentúa en la propia percepción como alguien indeseable o no merecedor de estima o perdón (Martínez et al., 2011).

Partiendo que el grupo casos se encontraba institucionalizado por el consumo de alguna sustancia (legal o ilegal), la frecuencia en el consumo, abuso y dependencia es superior al grupo control; en esta medida, hay diferencias significativas en torno a las creencias que mantienen la dependencia al consumo de sustancias. En las personas con abuso de sustancias psicoactivas predominan más las creencias relacionadas con el consumo que las creencias de autocontrol.

En concordancia con lo propuesto por (Beck et al., 1999), las creencias nucleares son el resultado de un grupo de creencias; las creencias nucleares interactúan con los estresores vitales del individuo, generan ansiedad, disforia e ira; "estas situaciones estresantes o estímulos no causan directamente el craving sino que activan las creencias relacionadas con la drogas que llevan al craving" (p.84).

Así pues, analizando la correlación en los grupos controles y casos, se detalla que las creencias acerca del consumo es un predictor a la dependencia de sustancias, interviniendo en esta predicción rasgos de depresión y ansiedad que presenten los individuos. 
A manera de conclusión, se considera de gran relevancia el estudio de las creencias en la problemática del consumo, el abuso y la dependencia de sustancias alcohólicas y no alcohólicas. La importancia de este estudio, radica en identificar no solo las creencias nucleares relacionadas con el consumo de sustancias que predisponen la dependencia a sustancias, sino también, tener presente indicadores clínicos como la depresión y la ansiedad. Es así, que los procesos de rehabilitación serían más eficaces si intervienen las variables cognitivas (creencias nucleares) asociadas a las adicciones, en relación con las variables clínicas de depresión y ansiedad en los pacientes que están en recuperación por la dependencia a sustancias psicoactivas; es este un gran reto que convoca no solo a los profesionales sino también a la instituciones.

Como limitaciones del estudio se hace referencia a las dificultades para acceder a la muestra, dadas las restricciones de los centros de rehabilitación. Finalmente, la investigación deja la puerta abierta para pensar futuros estudios relacionadas con las influencia de las creencias y la adherencia al tratamiento de sustancias psicoactivas.

\section{Referencias}

APA (2013). Diagnostic and Statistical Manual of Mental Disorders (DSM-V) (Fifth Edition). Washington, DC: American Psychiatric Association.

Beck, A., Wright, F., Newman, C., y Liese, B. (1999). Terapia cognitiva de las drogodependencias. Barcelona, España: Paidós.

Brecher, E.M. (1972). Licit and illicit drugs: The Consumers Union report on narcotics, stimulants, depressants, inhalants, hallucinogens and marijuana including caffeine, nicotine, and alcohol. Boston: Little and Brown.

Cameron, D.C. (1968). Youth and drugs. Journal of the American Medical Association, 206 (6), 1267-1271. doi:10.1001/jama.1968.03150060041008.

Delgado, D.M., Pérez, A., y Scoppetta, O. (2000). Consumo de drogas en Colombia: un análisis sofisticado de datos simples. Adicciones. 13(1), 81-88. doi: http:// dx.doi.org/10.20882/adicciones.588. 
Derogatis, L. R. (1983). The SCL-9O-R administration, scoring and procedures manual. Towson, MD: Clinical Psychometric Research.

Derogatis, L. R. (2002). SCL-9O-R. Cuestionario de 90 ítems. Adaptación española de J. L. González de Rivera et al. Madrid: TEA Ediciones.

García, R., Rodríguez, S., y Córdova, A. (2008). Factores motivacionales protectores de la depresión. Salud mental, 31(6), 453-459. Recuperado http://www.scielo. org.mx/scielo.php?script=sci_arttext\&pid=S0185-33252008000600005

Ministerio del Interior y de Justicia, Ministerio de la Protección Social y Dirección Nacional de Estupefacientes (2009). Estudio nacional de consumo de drogas en Colombia. República de Colombia, 2008. Bogotá: Editora Guadalupe

Martínez, I. et al. (2011). Categorías de creencias adictivas en pacientes de un programa de rehabilitación en farmacodependencia. Revista Española de Drogodependencias. 36(2), 185-196. Recuperado de http://www.aesed.com/descargas/revistas/v36n2_4.pdf

Martínez, J., Verdejo, A., y Becoña, E. (2012). Estudio exploratorio del impacto de las creencias nucleares relacionadas con la adicción en el consumo tras la abstinencia. Trastornos Adictivos. 14(4), 105-111. doi: 10.1016/S15750973(12)70053-3.

Ministerio de Salud y Colciencias. (2015). Encuesta Nacional de Salud Mental 2015. Recuperado de http://www.odc.gov.co/Portals/1/publicaciones/pdf/ consumo/estudios/nacionales/CO031102015-salud_mental_tomoI.pdf.

Organización Mundial de la Salud (2010). Estrategia Global para la Reducción del Consumo Nocivo de Alcohol. Suiza: Editorial de la OMS.

Sheehan, D.V., Lecrubier, Y. (2000). M.I.N.I. Mini internacional neuropsychiatric interview. Versión en Español. Madrid: Instituto IAP.

Su, C., Kung, C., Huang, M., Lee, W. (2011). A Study of Relationship among Drugused Impulsivity, Beliefs and Craving, and Intention to Relapse on Drug Addicts. Asian Journal of Psychiatry, 4(1), 58-65. Recuperado de http://www. asianjournalofpsychiatry.com/article/S1876-2018(11)60222-o/abstract. 
Radloff, L.S., Locke, B.Z. (1986). The Community Mental Health Assessment Survey and the CES-D scale. En: Weissman M, Myers J, Ross C, editors. Community surveys. New Brunswick, NJ: Rutgers University Press.

Torres, Y., Bareño, J., Sierra, G. (2012). Primer Estudio Poblacional de Salud Mental Medellín, 2011-2012. Medellín, Colombia: Universidad CES - Facultad de Medicina. 